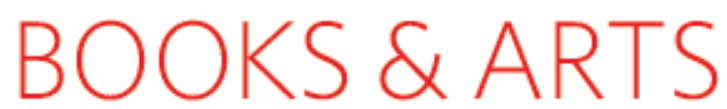

\title{
Expanding the Universe
}

\section{Just how many dimensions are there?}

Warped Passages: Unravelling the

Universe's Hidden Dimensions

by Lisa Randall

Allen Lane: 2005.512 pp. $€ 25$

To be published in the US in September by

Ecco (HarperCollins).

\section{Paul Davies}

In 1884 a curious little book appeared called Flatland. Written by Edwin Abbott, an English mathematician, it was destined to become a minor classic. It describes a world of twodimensional beings - entities of various shapes for whom 'up' and 'down' have no meaning. The book describes their baffling experiences when presented with threedimensional processes that penetrate their universe. Apart from its entertainment value, Flatland serves to educate the reader in the possibility that our three-dimensional world might in fact be merely a 'slice' or section in a higher-dimensional space to which access is restricted. That is, there might be a direction in space, which we can neither see nor move through, that points at right-angles to the familiar perpendicular directions (latitude, longitude and altitude). The basic message of Flatland is that just because we can't visualize higher dimensions, it doesn't mean they don't exist.

Mathematicians have long known that spaces of more than three dimensions make good logical and mathematical sense. Indeed, they are routinely used as a calculational device in science and engineering. In recent years, some theories of fundamental physics have postulated that additional dimensions of space really exist in the physical world. Warped Passages explains the rationale behind this seemingly extravagant claim.

There are two distinct ways in which one or more dimensions might be concealed from our senses. They could be rolled up to a very small size, just as a garden hose, which looks like a wiggly line from afar, turns out on closer inspection to be a two-dimensional sheet rolled into a thin tube. The idea that what we perceive as a point in space is in reality a tiny circle around a fourth dimension dates back to attempts in the 1920 s to provide a unified geometrical description of gravitation and electromagnetism based on Einstein's general theory of relativity. These days physicists are more ambitious and are trying to incorporate

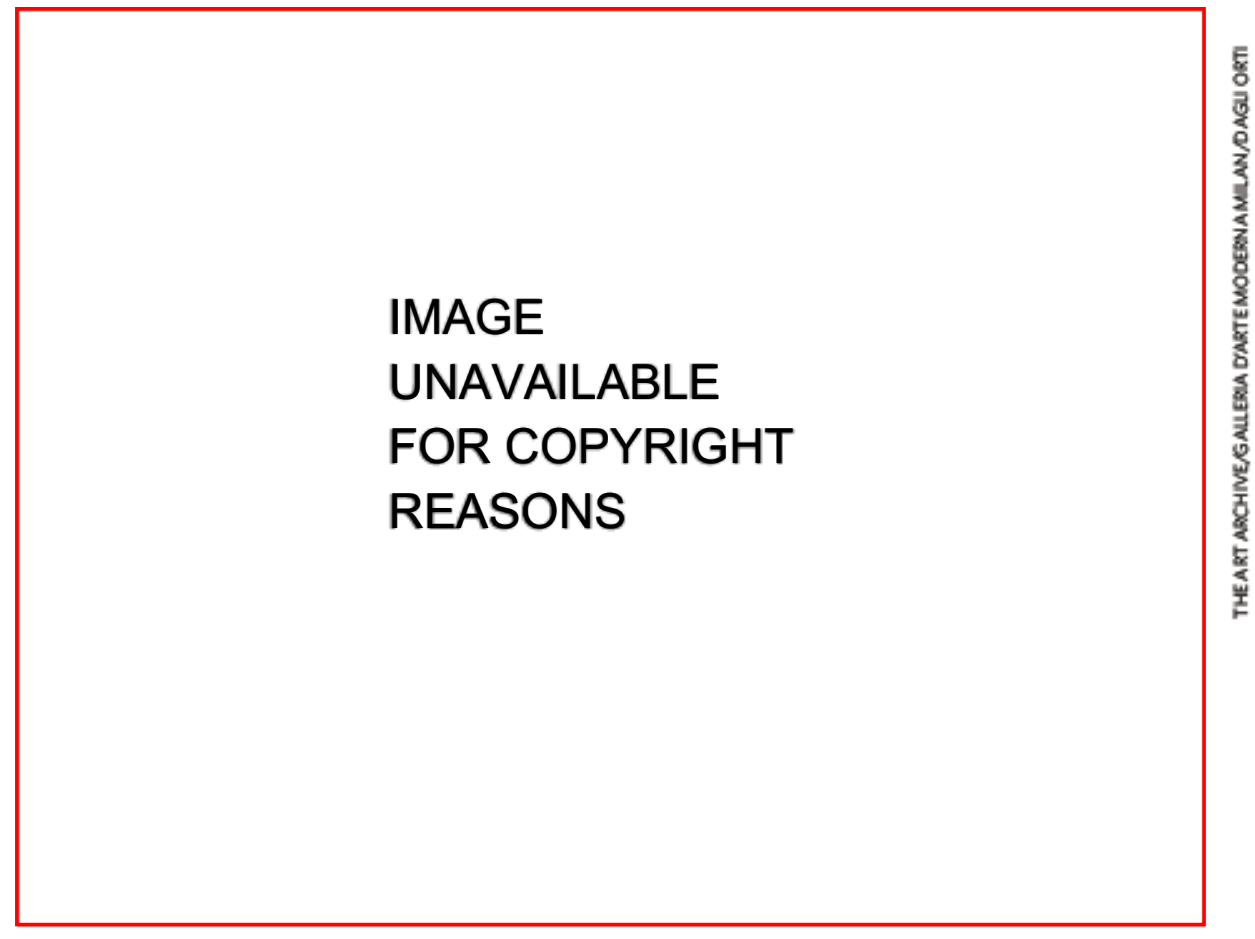

Like Jackson Pollock paintings, specialized science books can provide a rewarding cultural experience.

all the forces of nature, including the weak and strong nuclear forces, in a grand unified scheme. The most promising candidate, string theory, describes all matter as little loops of string that vibrate in ten-dimensional spacetime, of which six dimensions are rolled up in a complicated shape.

The other way to achieve hidden extra dimensions of space is to suppose that some sort of physical force barrier confines all normal matter, together with the light by which we see the world, to a three-dimensional 'membrane' embedded in a four-dimensional 'bulk'. The author of Warped Passages, Lisa Randall, is an expert in these 'brane' theories, which emerge naturally as by-products of string theory.

These outlandish ideas would be pointless speculation unless there was some way for the extra dimensions to manifest themselves. String theory predicts that at energies a billion billion times those attained by particle accelerators, observable things would happen to the strings in the rolled-up dimensions. Brane theory is less demanding; it has been conjectured that the Large Hadron Collider (LHC), currently being built at the CERN particlephysics laboratory near Geneva, might reveal traces of a fourth space dimension. This is because gravitation is not confined to branes but leaks out into the bulk. Some versions of the theory predict that, as a result, mini black holes and wormholes could conceivably be created from the high-speed collisions between the LHC's protons and antiprotons.

Randall has to confront an uncomfortable fact that faces most authors who tackle sweeping ideas of unification in fundamental physics. The book's peg - the possible existence of extra dimensions in space - is easy enough to explain. But motivating the conjecture requires a grand tour of some of the toughest and most abstract topics in science. To make her case for higher dimensions, Randall must first explain the general theory of relativity, with its notion of warped space-time. She then needs to set out the standard model of particle physics, with its confusing proliferation of subatomic objects - quarks, leptons, bosons of various sorts - and the details of the forces that act between them. Then she has to go into esoteric topics such as spontaneous symmetry breaking, Higgs potentials and supersymmetry. Because unification physics is formulated in terms of quantum mechanics - 
or rather, the quantum theory of fields, a much more demanding extension - that has to be covered too. Randall must do all this before she can properly describe string theory, and must then proceed to a more obscure generalization called M-theory. Furthermore, the exposition must be crafted without the explicit use of mathematics. Only then can Randall explain how branes emerge from a welter of concepts that are largely unfamiliar to the general reader. She achieves this tour de force by cutting quite a few corners, but even so, this is a long and dense read by any standards.

I suppose it is in the nature of unification that one can't leave anything out. After all, the thrust to create a final 'theory of everything' sprang from a desire to bring all physical theory into a common conceptual scheme. Randall makes a heroic attempt at completeness, but I wonder how many non-physicists will stay with the narrative to the bitter end.

A paradox of 'popular' science publishing is that even highly technical and specialized books still sell well, especially when there is a new author or a new twist to the subject that attracts headlines. I suspect that these books are rarely understood. Perhaps readers don't really intend to follow them studiously, but wade through the expositions as a cultural experience, rather like reflecting on a Jackson
Pollock painting - you know it's very clever and you assume it means something profound to the creator, but it belongs to an alien world. Maybe readers of popular physics and cosmology books simply enjoy being perplexed, or are satisfied merely to glimpse, voyeuristically, the arcane world of the theoretical physicist without actually having the foggiest idea of what is going on. Whatever it is that attracts these readers, Warped Passages has plenty of intriguing material to keep them happy.

Paul Daviesis at the Australian Centre for Astrobiology, Macquarie University, Sydney, New South Wales 2109, Australia. His latest book is How to Build a Time Machine.

\section{Making small talk}

\section{The Language of Life: How Cells Communicate in Health and Disease by Debra Niehoff \\ Joseph Henry Press: 2005. 306 pp. \$27.95}

\section{Fran Balkwill}

${ }^{\alpha}$ The language of all cellular societies is similarly based not on sounds or gestures but on chemistry. Using molecules where we would use words, constructing sentences from chains of proteins, the cells that make up...multicellular organisms inform, wheedle, command, exhort, reassure, nurture, criticize and instruct each other." This is how Debra Niehoff introduces The Language of Life, her original and absorbing book on the language of modern biology.

At its best, the prose is quite breathtaking. The first chapter immerses us in the world of a waltzing, shuffling, twirling Escherichia coli bacterium as it moves towards a potential meal. The process of chemotaxis is beautifully elucidated, with wonderful analogies to explain receptor-ligand interactions. Take for instance this personal ad: "Are you my better half?'-SFP (single folding protein) with secure position in healthy cell seeks compatible molecule with interest in chemical engineering, architecture, or communication for exclusive short-term relationship."

Niehoff excels when describing the chemical language of bacterial colonies, neurons, the immune system and the first eukaryotes. Indeed the 16 pages describing the innate and adaptive immune systems are an almost perfect example of how to communicate complex scientific ideas to a non-specialist audience.

Cell signalling is a complex subject, especially as it occurs in the cells of vertebrates, and the general reader may struggle in some sections of the book. However, after each 'tough' molecular-biology section, Niehoff glides easily into simple analogies, or even family anecdotes, and manages to keep a reader's interest. The descriptions of cell communication are enlivened by stories of key scientists and their discoveries, and by interviews with some of those currently contributing to the field. This important subject is moving quickly as scientists rapidly decipher the language of cells. It has a strong translational element and drives pharmaceutical research.

The 'disease' aspect of the title mainly relates to cancer and autoimmunity, where basic research has already led to novel, targeted and effective therapies. To cover all this ground, the book needs to be both wide-ranging and topi$\mathrm{cal}-$ and it is, impressively so. Niehoff has done her research well.

Biology is a continuum, but sometimes this point is not made strongly enough. Readers might benefit from more frequent reminders of the parallels between primitive and more sophisticated cellular systems. Although the final chapter on the 'virtual cell' is important, I would have appreciated a concluding chapter that brought the whole story together, emphasizing the common lines of communication

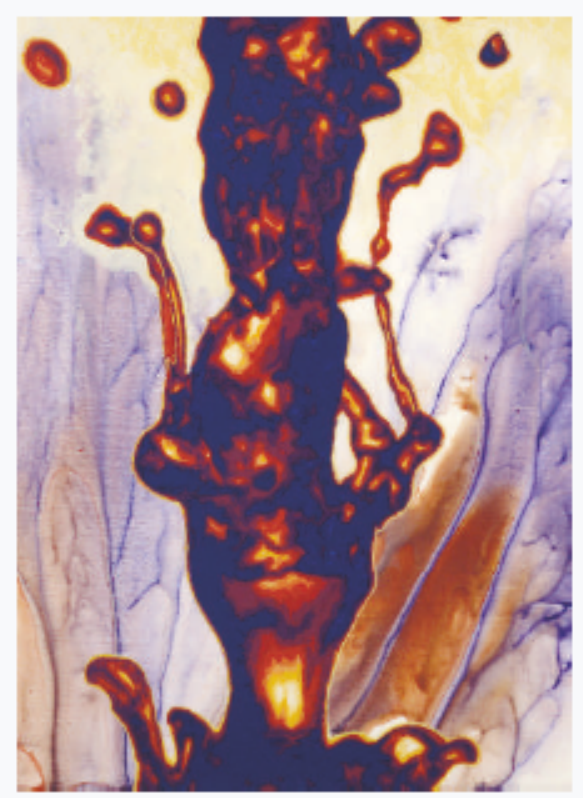

that linkhigher organisms with marine bacteria and the Hawaiian bobtail squid.

Having said that, this book is an invaluable introduction to, or reminder of, biomedical science for many lab scientists, and will also be enjoyed by a general scientific audience. Many Nature readers spend their working days trying to listen to the language of cells and to find ways to stop cells talking so much. Will these readers enjoy this book? My own personal benchmark for a 'popular' science book is that it is placed ahead of books by Ian Rankin, P. D. James and Patricia Cornwell as favourite bedtime reading. Niehoff's book passes that test. I shall certainly take it into the lab as recommended reading for my $\mathrm{PhD}$ students, and will be interested to hear their views. Fran Balkwill is at the Cancer Research UK Clinical Cancer Centre, Barts and the London, Queen Mary's School of Medicine and Dentistry, John Vane Science Centre, Charterhouse Square, London EC1M 6BQ, UK.

\section{EXHIBITION Colour vision}

"The freedom and responsibility of selecting a palette for an image is both mine and the original scientists'," says Jonathan Feldschuh, a painter based in New York who works with scientific images. His recent inspiration has come from simulations that visualize complex data, especially those representing phenomena that cannot be observed directly.

Scientists like to use colours objectively to represent information in such cases. Feldschuh also uses colour, to provide an aesthetic element, as in this image Drop Formation \#2. Its warm tones contrast with its shapes, which are reminiscent of nuclear explosions.

'Simulations', an exhibition of Feldschuh's work, can be seen at the Cynthia Broan Gallery in New York until 9 July. 